\title{
Microflora of Bile Aspirates in Patients With Acute Cholecystitis With or Without Cholelithiasis: A Tropical Experience
}

\author{
Malini R. Capoor ${ }^{1}$, Deepthi Nair ${ }^{1}$, Rajni' ${ }^{2}$, Geetika Khanna ${ }^{2}$, S.V. Krishna ${ }^{3}$, M.S. Chintamani ${ }^{3}$ and Pushpa Aggarwal ${ }^{2}$ \\ Department of ${ }^{1}$ Microbiology, ${ }^{2}$ Pathology and ${ }^{3}$ Surgery, Vardhman Mahaveer Medical College \& Safdarjung Hospital; New Delhi, India
}

\begin{abstract}
The current study determined the spectrum of biliary microflora with special emphasis on enteric fever organisms in patients with acute cholangitis with and without cholelithiasis or other biliary diseases. The patients were divided into three groups: Group A consisted of patients with acute cholecystitis with cholelithiasis; Group B consisted of patients with acute cholecystitis with gastrointestinal ailments requiring biliary drainage and group $\mathrm{C}$ consisted of patients with gallbladder carcinoma. Gallbladder, bile and gallstones were subjected to complete microbiological and histopathological examination. Antimicrobial susceptibility of the isolates was performed as per CLSI guidelines. Bacteria were recovered from 17 samples (32\%) in Group A, $17(51.4 \%)$ in Group B and 1 (1.6\%) in Group C. The most common organisms isolated were Escherichia coli $(11,29.7 \%)$, Klebsiella pneumoniae (10, $27 \%)$, Citrobacter freundii (3, 8.1\%), Salmonella enterica serovar Typhi (3, 8.1\%), etc. The majority of Enterobacteriaceae isolates were susceptible to piperacillin-tazobactam and meropenem. As regards Salmonella spp., S. Typhi was isolated from $2(3.8 \%)$ patients in Group A and $1(16 \%)$ in Group C. Antimicrobial susceptibility of potential causative organisms, the severity of the cholecystitis, and the local susceptibility pattern must be taken into consideration when prescribing drugs. A protocol regarding the management of such cases should be formulated based on observations of similar studies.
\end{abstract}

Key-Words: Bile, acute cholecystitis, Salmonella enretica serovar Typhi.

Acute cholecystitis is an infection of the biliary tract, which results from bile stasis due to chronic obstruction. The obstruction is usually attributed to gallstones in $80 \%$ of cases. The causes of acalculous cholecystitis include biliary structures, human immunodeficiency virus cholangiopathy, biliary parasites and primary sclerosing cholangitis. Other causes include complicated cases of burns, trauma, major surgery, diabetes and unusual bacterial infections of the gallbladder (Salmonella spp. or Vibrio cholerae) and other systemic infections (tuberculosis and syphilis) [1,2].

Biliary obstruction causes an increase in ductal pressure, resulting in bacterial proliferation and dissemination. Bacterial infection is the most common type of acute cholangitis, with a Gram-negative preponderance. Gram-positive and anaerobic are uncommon causative agents. Viral and fungal agents are rare [1,2].

In an enteric fever endemic country like India, Salmonella enterica serovar Typhi and S. Paratyphi A are among the major biliary pathogens. Enteric fever persists for many years after convalescence and increases the risk of hepatobiliary malignancy [3-9]. Even with prompt diagnosis and treatment acute cholangitis can lead to septicemia and complications like emphysema, gangrene, perforation and chronic cholecystitis [2].

The current study was undertaken to determine the spectrum of biliary microflora with special emphasis on enteric fever organisms in patients with acute cholangitis with or without cholelithiasis or other biliary diseases.

Received on 2 December 2007; revised 7 March 2008.

Address for correspondence: Dr Deepthi Nair. D II, 2201, Vasant Kunj, New Delhi-110064. E-mail: deepthinair2@gmail.com.

The Brazilian Journal of Infectious Diseases 2008;12(3):222-225. (C) 2008 by The Brazilian Journal of Infectious Diseases and Contexto Publishing. All rights reserved.

\section{Material and Methods}

One-hundred and four cases of biliary diseases were diagnosed and treated in the department of Microbiology and Surgery at Vardhman Mahavir Medical College and Safdarjung Hospital, New Delhi, over a period of three years (2005-2007). For the final analysis, the bile samples were divided into three groups. Group A consisted of patients with acute cholecystitis with cholelithiasis; Group B consisted of patients with acute cholecystitis with gastrointestinal ailments requiring biliary drainage and group $\mathrm{C}$ consisted of patients with gallbladder carcinoma. For Group B patients, the bile aspirates were received from surgery, pediatrics and the ICU (intensive care unit). The demographic profile and clinical examination of these patients were noted in a performa. Routine examination of hemogram, urine and serum biochemistry, and chest X-Ray were done.

An ultrasonogram of the upper abdomen was carried out to identify the site of obstruction, number of stones and condition of gallbladder.

Acute cholecystitis was defined by triad of right upper quadrant tenderness, fever and leukocytosis. Serum bilirubin and liver enzyme showed modest elevation.

Stool samples of these patients were collected preoperatively.

For Group A patients, laparotomy cholecystectomy or open cholecystectomy was performed as per patient condition. During open or lap-cholecystectomy, the gallbladder was excised and sent to the microbiology laboratory in normal saline. Bile was aspirated during cholecystectomy and sent in a sterile container.

Gallbladder, bile and gallstones were subjected to complete microbiological and histopathological examination. All the samples were processed as per standard procedures. In brief gallbladder tissue was punched from six different 
sites and gallstones were crushed with sterile rod and blade. Tissue samples, gallstones and bile aspirate were directly inoculated onto various bacteriological media. These were also processed further in selective (selenite- $F$ broth) and non-selective (brain heart infusion) broth. The isolates were identified by routine biochemical tests and were confirmed by serotyping, wherever indicated.

Antimicrobial susceptibility of the isolates was performed as per CLSI guidelines [10]. For Gram-negative isolates the following antimicrobials were used: ampicillin $(10 \mu \mathrm{g})$, chloramphenicol (30 $\mu \mathrm{g})$, co-trimoxazole $(1.25 / 23.75 \mu \mathrm{g})$, nalidixic acid (30 $\mu \mathrm{g})$, ciprofloxacin (5 $\mu \mathrm{g})$, amikacin (30 $\mu \mathrm{g})$, ceftazidime (30 $\mu \mathrm{g})$, netilmycin (30 $\mu \mathrm{g})$, piperacillin $(100 \mu \mathrm{g})$, piperacillin/tazobactam $(100 / 10 \mu \mathrm{g})$, meropenem $(10 \mu \mathrm{g})$. For Gram-positive isolates: oxacillin, penicillin $(10 \mu \mathrm{g})$, vancomycin (30 $\mu \mathrm{g})$, erythromycin (15 $\mu \mathrm{g})$, ciprofloxacin (5 $\mu \mathrm{g})$ and gentamicin $(10 \mu \mathrm{g})$.

\section{Results}

The age of the patients in the study ranged from 13 years to 72 years (median $=41$ years). The male female ratio in the study was 0.48 . Group A consisted of 53 patients, Group B consisted of 45 patients and Group C consisted of 6 patients. The most common presenting complaint in all the groups was right upper abdomen pain. Group B patients with other biliary tract diseases included cases of chronic pancreatitis, gallbladder anomalies and liver disorders.

From a total of 104 bile samples, bacteria were isolated in 37 samples (35.6\%). Monomicrobial infection was observed in 32 (30.8\%). Polymicrobial infection was seen in 5 (4.8\%). The most common organisms isolated were Escherichia coli (11, 29.7\%), Klebsiella pneumoniae (10, 27\%), Citrobacter freundii (3, 8.1\%), Salmonella enterica serovar Typhi (3, 8.1\%), Pseudomonas aeruginosa (2, 5.4\%), Acinetobacter spp. (1, 2.7\%), Candida krusei (1, 2.7\%), Staphylococcus aureus (1, 2.7\%). Polymicrobial infection of $P$. aeruginosa with $K$. pneumoniae was observed in 4 patients (3.8\%).
Table 1 depicts the distribution of various microorganisms in the three groups.

Bacteria were recovered from 17 samples (32\%) in Group A, 17 (51.4\%) samples in Group B and 1 (1.6\%) in Group C. As regards Salmonella spp., S. Typhi was isolated in 2 patients (3.8\%) in Group A, 1 (16\%) in Group C and none in Group B. Microbial cultures of gallbladder tissue were superior followed by bile and gallstones as regards bacterial yield (17 vs.15 each). Contamination was most commonly seen in bile aspirate cultures. For Group A patients histopathological evidence was consistent with the findings of acute cholecystitis with acute cholelithiasis. For Group C patients histopathological examination showed adenocarcinoma in 5 cases and showed atypical changes suggestive of malignancy in a single case. For Group A patients, laparotomy cholecystectomy was performed in 31 cases (58.5\%) and open cholecystectomy was performed in 22 cases (41.5\%).

No disparity was observed between patients undergoing these two procedures as regards the morbidity associated with postoperative period.

\section{Discussion}

In this study the median age of the patients was 41 years and the male: female ratio was 0.48 . Similar findings have been observed in prior studies [3].

Significant numbers of patients in Group B with other biliary diseases had culture positive bile (51.4\%) as compared to patients in Group A with acute cholecystitis with cholelithiasis (32\%). This may be attributed to history of prior biliary surgery in the majority of patients (>80\%) of Group B. As many of the organisms that can colonize the T-tube are introduced into a sterile biliary tract during surgery, possibly from the gallbladder wall during surgical manipulation $[2,3]$. Furthermore, it was also observed in this study that known multi-drug resistant gastrointestinal organisms such as $P$. aeruginosa, Acinetobacter spp., and $S$. aureus were more frequently observed in Group B patients. Polymicrobial

Table 1. Distribution of various microorganisms among different groups.

\begin{tabular}{lcccc}
\hline Microorganism monomicrobial & $\begin{array}{c}\text { GroupA } \\
\mathbf{n - 5 3}\end{array}$ & $\begin{array}{c}\text { Group B } \\
\mathbf{n = 6}\end{array}$ & $\begin{array}{c}\text { Group B } \\
\mathbf{n = 4 5}\end{array}$ & $\begin{array}{c}\text { Group C } \\
\mathbf{n = 6}\end{array}$ \\
\hline E. coli & 7 & - & 4 & - \\
K. pneumoniae & 4 & - & 6 & - \\
S. Typhi & 2 & 1 & - & 1 \\
C. freundii & 3 & - & - & - \\
P. aeruginosa & - & - & 2 & - \\
Acinetobacter spp. & 1 & - & - & - \\
Staph. aureus & - & - & 1 & - \\
Can. kruseii & - & - & 1 & - \\
Polymicrobial & - & - & 4 & - \\
P. aeruginosa with K. pneumoniae & - & - & 1 & - \\
P. aeruginosa with E. coli & - & &
\end{tabular}


Table 2 Antimicrobial** susceptibility of isolates in the study

\begin{tabular}{lccccccccc}
\hline Isolate (N)* & A & Pc & Ci & Ca & Cf & Nt & Ak & Pc/Tz & M \\
\hline E. coli (12) & & 3 & 3 & - & 4 & 7 & 8 & 12 & 12 \\
K. pneumoniae (14) & 1 & 9 & 9 & - & 8 & 6 & 7 & 14 & 14 \\
S. Typhi (3) & 3 & - & 3 & - & 3 & - & - & - & - \\
C. freundii (3) & 1 & 2 & 2 & 2 & 2 & 2 & 2 & 3 & 3 \\
P. aeruginosa (7) & - & 2 & - & 2 & 2 & 4 & 4 & 6 & 6 \\
Acinetobacter spp. (1) & - & 1 & - & 1 & 1 & 1 & 1 & 1 & 1 \\
\hline
\end{tabular}

*Staphylococcus aureus (1) sensitive to oxacillin, erythromycin, penicillin vancomycin, ciprofloxacin, gentamicin; *All S. Typhi were sensitive to ampicillin, chloramphenicol, co-trimoxazole and nalidixic acid; ${ }^{*} C$. krusei was sensitive to amphotericin, but resistant to fluconazole; **Antimicrobials: A=ampicillin; $\mathrm{Pc}=$ piperacillin; $\mathrm{Ci}=$ ceftriaxone; $\mathrm{Ca}=$ ceftazidime; $\mathrm{Cf}=$ ciprofloxacin; $\mathrm{Nt}=$ netilmycin; Ak=amikacin; Pc/Tz=piperacillin/tazobactam; M=meropenem.

infection was only observed in this group. These were all hospital acquired infections as these occurred $>48$ hours after admission to hospital and the patient was not incubating the infection at the time of admission. Polymicrobial infection has been previously reported in bile aspirates $[3,11,12]$. The most frequent cause of monomicrobial infection was $E$. coli, followed by other members of the family enterobacteriaceae, which form the gut-microflora. This has been reported in prior studies [12-14]. Acute supportive cholangitis and cholangiohepatitis occur due to the presence of bacteria which originate in the gut and ascend to the lower bile duct due to various predisposing conditions (biliary stasis, cholelithiasis, chronic pancreatitis, irritable bowel disease, biliary strictures, anatomic abnormalities, etc). Superinfection is a common complication as these patients undergo repeated episodes and visit hospital during severe attacks. Moreover, calculi are known to induce stasis, promoting chronic infection leading to increased turnover of primary bile acids to secondary bile acids, which are tumor promoters and initiators [9]. It is documented in literature that gallbladder carriage of S. Typhi and S. Paratyphi A increases the risk of hepatobiliary carcinoma 8.47 times [4]. In our study the prevalence (percentage positivity) of $S$. Typhi was $2.8 \%$ in gallbladder disease (Group A and Group C) patients. Nonetheless, prevalence of $S$. Typhi in cholelithiasis patients (Group A) and gallbladder carcinoma could not be compared as the sample size of Group $C(n=6)$ was smaller than that of Group A ( $n=53$ ). In literature other workers have observed the prevalence of $S$. Typhi in bile of cholelithiasis patients from 1 to $34 \%[3,7,15]$ and for that of gallbladder carcinoma patients from 1 to $40 \%[6,7,15]$. The similar figure for other biliary diseases was $1.6 \%$ [3] in a study. However, in contrast to this, for Group B patients we could not find association of S. Typhi or other Salmonellae.

In our study stool samples of all the patients were not analyzed, as fecal carriage does not give a true figure of carrier incidence due to intermittent shedding and there is also a requirement to carry out several examinations before carriage could be excluded. Therefore, stool cultures of only those patients which showed biliary carriage with $S$. Typhi were performed.
A naerobes are infrequent causes of biliary infections and are usually associated with chronic cholecystitis [1]. Therefore, with this in mind, anaerobic culture was not performed in the current study which was primarily done on acute cholecystitis patients. In the current study, patients were initiated on metronidazole to avoid postoperative anaerobic infection.

A single isolate of Candida krusei was isolated from the bile culture of a 65 year old female with gallbladder carcinoma in ICU. The risk factors attributed were $>30$ days stay in ICU, ventilator and broad spectrum antimicrobials ( $>30$ days). Candida cholecystitis is a rare disease and most reported cases have been isolated from patients with immunosuppression, on broad spectrum antimicrobials and following invasive procedures of the biliary tract [16].

In this study, the mortality was $0.9 \%$. A single patient of gallbladder carcinoma with $C$. krusei expired due to the aforementioned predisposing factors. In acute cholecystitis mortality is seen in around 5\% of cases. It is attributed to comorbid conditions associated with the disease such as malignancy, age $>80$ years etc [1].

Although surgical intervention remains the mainstay of therapy for acute cholecystitis and its complications, a period of hospital stay is required before elective or emergency cholecystectomy. In the current study, the empirical antibiotics were prescribed as per recommended guidelines $[17,18]$ and these were changed as per antimicrobial susceptibility results. As postoperative complication of wound infection, abscess formation or sepsis are reduced in antibiotic treated patients. In brief, for mild cases of biliary colic, the administration of non-steroidal anti-inflammatory drugs (NSAIDS) is recommended to prevent progression of inflammation (recommendation grade A). For moderate infection, agents with a narrow spectrum of activity such as cefuroxime or ciprofloxacin plus metronidazole are preferred. For severe infections, combination drugs or carbapenem are recommended. The latter two also required hydration and electrolyte correction and elimination of oral intake.

In our series of patients, barring a single isolate of $P$. Aeruginosa, the majority of isolates were susceptible to piperacillin-tazobactam and meropenem. Prior studies 
observed excellent responses with these drugs with quinolones for Gram-negative isolates and vancomycin for Gram-positive isolates being preferred [13,15,19]. As regards, $S$. Typhi, these were all susceptible to ciprofloxacin and ceftriaxone. This is despite the fact that there are increasing reports of resistance to these drugs from the Indian subcontinent $[20,21]$. Therefore, antimicrobial activity against potential causative organisms, the severity of the cholecystitis, and the local susceptibility pattern must be taken into consideration when prescribing drugs.

The majority of hospitals, in tropical countries cannot perform bile cultures due to a lack of facilities for biliary drainage. A protocol regarding the management of such cases should be formulated based on observations of similar studies. Nonetheless, many large scale studies are warranted in order to formulate guidelines in this regard.

\section{Reference}

1. Drugs and Therapeutics Bulletin 2005;43:62-4.

2. Greenberger N.J., Isselbacher K.J. Diseases of the gallbladder and bile ducts. In: Fauci S.A., Braunwald E., Isselbacher K.J., et al.(eds). Harrison's Principles of Internal Medicine. Mc GrawHill, New York, 1998.

3. Vaishnavi C., Singh S., Kochhar R. Prevalence of Salmonella enterica serovar Typhi in bile and stool of patients with biliary diseases and those requiring biliary drainage for other purposes. Jpn J Infect Dis 2005;58:363-5.

4. Prouty A.M., Schwesinger W.H., Gunn J.S. Biofilm formation and interaction with surfaces of gallstones by Salmonella. Infect Immunity 2002;70:2640-9.

5. Nath G., Singh H., Shukla V.K. Chronic typhoid carriage and carcinoma of the gall bladder. Eur J Cancer Prev 1997;6:557-9.

6. Dutta U., Garg P.K., Kumar R., Tandon R.K. Typhoid carriers among patients with gallstones are at increased risk for carcinoma of the gall bladder. Am J Gastroenterol 2000;95:784-7.

7. El-Zayadi A., Ghoneim M., Kabil S.M., et al. Bile duct carcinoma in Egypt: possible etiological factors. Hepatogastroenterol 1991;38:337-40.
8. Shukla V.K., Singh H., Pandey M., et al. Carcinoma of the gall bladder. Is it a sequel of typhoid. Dig Dis Sci 2000;45:900-3.

9. Sharma V., Chauhan V.K., Nath G., Kumar A., Shukla V.K. Role of bile bacteria in gall bladder carcinoma. Hepatogastroenterol 2007;54:1622-5.

10. CLSI. Performance standards for antimicrobial susceptibility testing, $17^{\text {th }}$ Informational Supplement. CLSI Document M100S16, 26 Wayne PA: CLSI, 2007.

11. Csendes A., Mitru N., Maluenda F., et al. Counts of bacteria and pyocites of choledochal bile in controls and in patients with gallstones or common bile duct stones with or without acute cholangitis. Hepatogastroenterol 1996;43:800-6.

12. Grizaz S., Stakyte M., Kincius M., et al. Etiology of bile infection and its association with postoperative complications following pancreatoduodenectomy. Medicina 2005;41:386-91.

13. Neve R. Biswas S., Dhir V., et al. Bile cultures and sensitivity patterns in malignant obstructive jaundice. Indian J Gastroenterol 2003;22:16-8.

14. Shimada K., Inamatsu T., Yamashiro M. Anaerobic bacteria in biliary disease in elderly patients. J Infect Dis 1977;135: 850-4.

15. Sattar I., Aziz A., Rasul S., Mehmood Z., Khan A. Frequency of infection in cholelithiasis. J Coll Physicians Surg Pak 2007; $17: 48-50$

16. Rodriguez V.E., Freuler C.B., Ezcurra C., Durlach R.A. Acute cholecystitis and biliary tract infections due to Candida. Rev Iberoam Micol 2007;24:152-4.

17. Yoshida M., Takada T., Kawarada Y., et al. Antimicrobial therapy for acute cholecystitis: Tokyo Guidelines. J Hepatobiliary Pancreat Surg 2007;14:83-90.

18. Solomkin J.S., Mazuski J.E., Baron E.J., et al. Guidelines for the selection of anti-infective agents for complicated intraabdominal infections. Clin Infec Dis 2002;37:997-1005.

19. Rerknimitr R., Fogel E.L., Kalayci C., Esber E., Lehman G.A., Sherman S. Microbiology of bile in patients with and without plastix biliary endoprosthesis. Gastrointest Endosc 2002;56:885-9.

20. Capoor M.R., Rawat D., Nair D., et al. In vitro activity of azithromycin, newer quinolones and cephalosporins in ciprofloxacin resistant Salmonella causing enteric fever. J Med Microbiol 2007;58:1490-4.

21. Saha S.K., Darmstadt G.L., Baqui A.H., et al. Molecular basis of resistance displayed by highly ciprofloxacin resistant Salmonella enterica serovar Typhi in Bangladesh. J Clin Microbiol 2006;44:3811-3. 